\title{
Determination of the Crystal Structure of Gamma-Alumina by Electron Diffraction and Electron Energy-Loss Spectroscopy
}

Henry O. Ayoola ${ }^{1 *}$, Cecile S. Bonifacio ${ }^{1}$, Matthew T. Curnan ${ }^{1,4}$, Stephen D. House ${ }^{1}$, Meng Li $^{1}$, Joshua $\mathrm{Kas}^{2}$, John J. Rehr ${ }^{2}$, Eric A. Stach ${ }^{3}$, Wissam A. Saidi ${ }^{4}$, and Judith C. Yang ${ }^{1,5}$

1. Dept. of Chemical and Petroleum Eng., University of Pittsburgh, Pittsburgh, PA (USA)

2. Dept. of Physics, University of Washington, Seattle, WA (USA)

3. Center for Functional Materials, Brookhaven National Laboratory, Upton, NY (USA)

4. Dept. of Mechanical Eng. and Materials Science, University of Pittsburgh, Pittsburgh, PA (USA)

5. Dept. of Physics and Astronomy, University of Pittsburgh, Pittsburgh, PA (USA)

*Corresponding author: hea23@pitt.edu

Gamma-alumina $\left(\gamma-\mathrm{Al}_{2} \mathrm{O}_{3}\right)$ is a metastable phase of $\mathrm{Al}_{2} \mathrm{O}_{3}$ with wide-ranging applications in fields such as catalysis due to its high surface area-to-volume ratio and surface properties. Yet despite heavy interest in and study of $\gamma-\mathrm{Al}_{2} \mathrm{O}_{3}$, its true crystal structure is still debated. $\gamma-\mathrm{Al}_{2} \mathrm{O}_{3}$ is traditionally described as having a spinel structure, but with cation vacancies added to maintain the correct stoichiometry. However, many different spinel and nonspinel structures for $\gamma-\mathrm{Al}_{2} \mathrm{O}_{3}$ have since been proposed. This is a problem for theoretical simulations of systems containing $\gamma-\mathrm{Al}_{2} \mathrm{O}_{3}$, since a model of $\gamma-\mathrm{Al}_{2} \mathrm{O}_{3}$ must be selected from the many models described in the literature. At present, the choice of model is often based on computational considerations rather than the accuracy of the model to experiment. Thus, clarification is needed on the accuracy of the existing models, and if possible, what the true structure of $\gamma-\mathrm{Al}_{2} \mathrm{O}_{3}$ is.

A major contributing factor to the uncertainty surrounding the structure of $\gamma-\mathrm{Al}_{2} \mathrm{O}_{3}$ is the heterogeneity of commercially available $\gamma-\mathrm{Al}_{2} \mathrm{O}_{3}$. To mitigate this, we synthesized near-single-crystal (SC) $\gamma-\mathrm{Al}_{2} \mathrm{O}_{3}$ thin films by controlled oxidation of single-crystal NiAl (110) and characterized these films using TEM, selected-area electron diffraction (SAED), and electron energy-loss spectroscopy (EELS). Through correlating these experimental measurements with simulated electron diffraction and EELS, we gauged the accuracy of the most commonly cited $\gamma-\mathrm{Al}_{2} \mathrm{O}_{3}$ models. We considered the following $\gamma-\mathrm{Al}_{2} \mathrm{O}_{3}$ models: Smrcok cubic spinel [1], Paglia tetragonal nonspinel [2], Digne monoclinic nonspinel [3], and the Pinto monoclinic spinel [4].

From the near-SC $\gamma-\mathrm{Al}_{2} \mathrm{O}_{3} \sim 80 \mathrm{~nm}$ thin film, a cross-sectional TEM sample was prepared using focused ion beam (FIB). A polycrystalline $\gamma-\mathrm{Al}_{2} \mathrm{O}_{3}$ thin film was also synthesized, by extending the growth duration, and transferred to a carbon-coated TEM grid. SAED was acquired from both samples, producing spot patterns from the near-SC film and ring patterns (e.g., Figure 1a) from the polycrystalline film. The ring pattern was converted to the line profile by azimuthally averaging the intensity of the rings and is plotted in Figure $1 \mathrm{~b}$ along with the equivalent simulated diffraction profiles for each model. Comparison of peak positions shows that the Digne model, arguably the most commonly used model in theoretical studies (by citation numbers), is the least accurate model. Furthermore, comparison of peak intensities suggests that the spinel models are more accurate than the Paglia nonspinel model. We are now working to determine the distribution of $\mathrm{Al}$ cations in $\gamma-\mathrm{Al}_{2} \mathrm{O}_{3}$ based on our data.

While SAED provides information on the unit cell dimensions and atomic distribution in crystallographic sites, EELS provides complimentary information about chemical state (and thus binding) by probing the electronic structure. $\mathrm{Al} \mathrm{L} 2,3$ and $\mathrm{O}$ K edge core-loss EELS spectra were acquired from the $\mathrm{SC}_{\gamma}-\mathrm{Al}_{2} \mathrm{O}_{3}$ film 
and shown in Figure 2. Near-edge EELS spectra were simulated for each model using the FEFF9 code [5]. Comparing the EELS spectra reveals that the nearest-neighbor coordination around $\mathrm{O}$ atoms for the Paglia model is significantly different from the coordination from the experimental EELS, while the other models are not easily distinguished at the energy resolution achieved in our experiments. We are now preparing experiments on higher energy resolution instruments to further discern between the models.

\section{References:}

[1] L. Smrcok, V. Langer and J. Krestan, Acta Crystallographica Section C, 62(2006), p. i83-i84.

[2] G. Paglia et al, Physical Review B, 68(2003), 144110.

[3] M. Digne et al, Journal of Catalysis 226(2004), p. 54-68.

[4] H.P. Pinto, R.M. Nieminen and S.D. Elliott, Physical Review B, 70(2004), 125402.

[5] K. Jorissen, J.J. Rehr and J. Verbeeck, Physical Review B, 81(2010), 155108.

[6] The authors acknowledge support from NSF CHE-1300544, CHE-1534630 and DOE-BES (DE-FG0297ER45623). WAS acknowledges start-up funding from the Mechanical Eng. and Materials Science Dept. at U. Pittsburgh. The experimental data was acquired using instruments at the Nanoscale Fabrication and Characterization Facility (NFCF) at U. Pitt, the AMCaT Lab at U.C. Davis, and the Center for Functional Nanomaterials, which is a U.S. DOE Office of Science Facility, at Brookhaven National Laboratory under Contract No. DE-SC0012704.
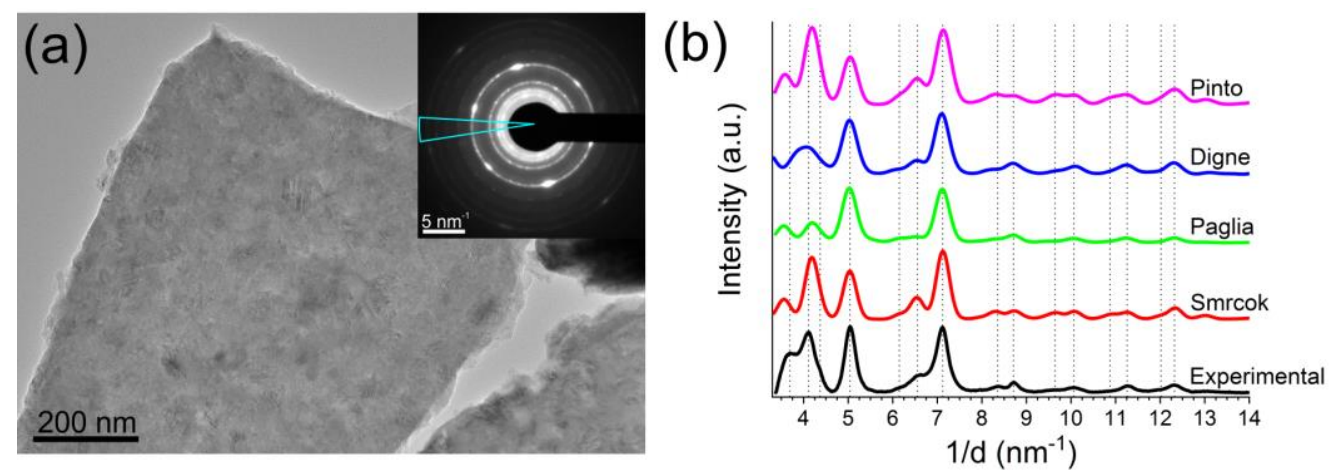

Figure 1. (a) TEM image of polycrystalline $\gamma-\mathrm{Al}_{2} \mathrm{O}_{3}$ film with inset SAED pattern. The blue wedge shows the area azimuthally averaged to produce the "Experimental" profile in (b) to avoid texture spots. (b) Comparison of experimental to simulated diffraction from the considered models, showing that all models are close to experiment, but the Smrcok cubic spinel model is the best match.
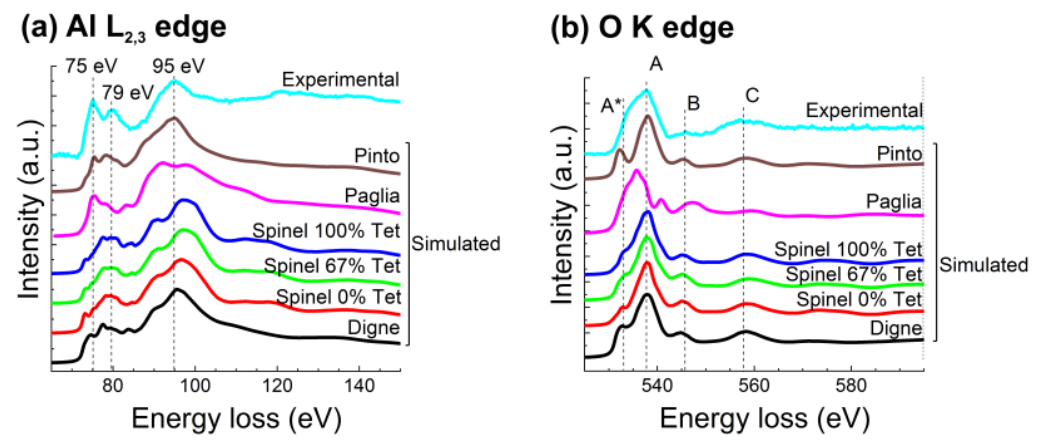

Figure 2. Comparison of experimental and simulated EELS Al L2,3 edge (a) and $\mathrm{O} K$ edge (b) spectra. The Al atoms in tetrahedral (Tet) positions in the cubic spinel model were varied in $\%$ occupancy relative to octahedral positions to cover the reported distributions in literature and compared with the tetragonal and monoclinic nonspinel models. For the $\mathrm{O} \mathrm{K}$ edge spectra, that of the tetragonal model differs significantly from those of the experimental and other simulated models. 